\title{
Connexin 43 expressed in endothelial cells modulates monocyte-endothelial adhesion by regulating cell adhesion proteins
}

\author{
DONGDONG YUAN*, GUOLIANG SUN*, RUI ZHANG*, CHENFANG LUO, \\ MIAN GE, GANGJIAN LUO and ZIQING HEI \\ Department of Anesthesiology, The Third Affiliated Hospital of Sun Yat-Sen University, \\ Guangzhou, Guangdong 510630, P.R. China
}

Received November 26, 2014; Accepted August 4, 2015

DOI: $10.3892 / \mathrm{mmr} .2015 .4273$

\begin{abstract}
Adhesion between circulating monocytes and vascular endothelial cells is a key initiator of atherosclerosis. In our previous studies, it was demonstrated that the expression of connexin $(\mathrm{Cx}) 43$ in monocytes modulates cell adhesion, however, the effects of the expression of $\mathrm{Cx} 43$ in endothelial cells remains to be elucidated. Therefore, the present study investigated the role of the expression of $\mathrm{Cx} 43$ in endothelial cells in the process of cell adhesion. A total of four different methods with distinct mechanisms were used to change the function and expression of $\mathrm{Cx} 43$ channels in human umbilical vein endothelial cells: $\mathrm{Cx} 43$ channel inhibitor (oleamide), enhancer (retinoic acid), overexpression of Cx43 by transfection with pcDNA-Cx43 and knock-down of the expression of $\mathrm{Cx} 43$ by small interfering RNA against $\mathrm{Cx} 43$. The results indicated that the upregulation of the expression of $\mathrm{Cx} 43$ enhanced monocyte-endothelial adhesion and this was markedly decreased by downregulation of $\mathrm{Cx} 43$. This mechanism was associated with $\mathrm{Cx} 43$-induced expression of vascular cell adhesion molecule-1 and intercellular cell adhesion molecule-1. The effects of $\mathrm{Cx} 43$ in endothelial cells was independent of $\mathrm{Cx} 37$ or $\mathrm{Cx} 40$. These experiments suggested that local regulation of endothelial $\mathrm{Cx} 43$ expression within the vasculature regulates monocyte-endothelial adhesion, a critical event in the development of atherosclerosis and other
\end{abstract}

Correspondence to: Dr Gangjian Luo or Professor Ziqing Hei, Department of Anesthesiology, The Third Affiliated Hospital of Sun Yat-Sen University, 600 Tianhe Road, Guangzhou, Guangdong 510630, P.R. China

E-mail: 18922102871@163.com

E-mail: heiziqing@sina.com

"Contributed equally

Key words: connexin 43, atherosclerosis, monocyte-endothelial adhesion, vascular cell adhesion molecule-1, intercellular cell adhesion molecule-1 inflammatory pathologies, with baseline adhesion set by the expression of $\mathrm{Cx} 43$. This balance may be crucial in controlling leukocyte involvement in inflammatory cascades.

\section{Introduction}

Atherosclerosis is the major cause of mortality in contemporary society (1). It is a chronic inflammatory disease with a complicated pathogenesis. Numerous risk factors are involved, which ultimately enhance the adherence of circulating monocytes to vascular endothelial cells, which are inflamed, damaged or exposed to turbulent shear-stress (2). This monocyte-endothelial adherence is considered to be the primary initiator of atherosclerosis (3). Adherent monocytes transmigrate into the arterial intima where they propagate, mature and accumulate lipids. These intimal monocytes transform into macrophage foam cells, a hallmark of atherosclerosis (4). In addition, adherent monocytes damage the vascular endothelium, causing the release of chemoattractants and inflammatory factors (5). The inflammatory reactions are therefore, self-reinforcing, eventually resulting in the formation of the atherosclerotic plaque (6). Therefore, monocyte-endothelial adhesion not only initiates the pathological process of atherosclerosis, but enhances its progression. Therefore, it is important to understand the mechanism of this adhesion and the factors, which control it.

A number of factors facilitate this initial step, including hemodynamic turbulence, secretion of inflammatory factors and dysfunction of the endothelial cells (7). Previous studies have indicated roles for connexin (Cx) hemichannels and gap junctions in the pathology of atherosclerosis $(8,9)$.

Connexins are a large family of proteins, which form hemichannels or gap junctions to promote transmembrane and intercellular coordination of tissue activity. Previous studies have indicated that connexins are involved in atherogenesis (8-12). There are three predominant connexins expressed in endothelial cells, Cx37, Cx40 and Cx43. Changes in their expression pattern have been assessed in cell lines and different animal models of atherosclerosis (9). Cx37 is considered to be atheroprotective. It was reported that $\mathrm{Cx} 37$ hemichannels regulate the initiation of atherosclerosis by inhibiting autocrine ATP-dependent modulation of monocyte-endothelial adhesion (8). Deletion of 
Cx40 promotes monocyte-endothelial adhesion and accelerates atherosclerosis, which is associated with gap junctions composed of Cx40 propagating adenosine-evoked anti-inflammatory signals (9). In our previous study, it was determined that the protective effects of $\mathrm{Cx} 43$ in cell adhesion of monocytes may be regulated by $\mathrm{Cx} 43$-stimulated ATP release (11). However, the function of the expression of $\mathrm{Cx} 43$ in endothelial cells remains to be elucidated.

Cx43 is the most widespread $\mathrm{Cx}$ in the cardiovascular system and is involved in normal physiology and several cardiovascular pathologies, including atherosclerosis (11). Cx43 is upregulated in human atheromatous plaque and in certain animal models of atherosclerosis. It enhances monocyte intimal migration, proliferation and apoptosis $(12,13)$. In the present study, the effects of $\mathrm{Cx} 43$ expression in human umbilical vein endothelial cells (HUVEC) on monocyte-endothelial adhesion were investigated.

\section{Materials and methods}

Cell lines and cell culture. HUVEC were enzymatically isolated from fresh umbilical cord, as previously described (11). The HUVEC were cultured in human endothelial-SFM medium (Invitrogen Life Technologies, Carlsbad, CA, USA), containing 15\% fetal bovine serum (Invitrogen Life Technologies), $100 \mathrm{U} / \mathrm{ml}$ penicillin-streptomycin (Invitrogen Life Technologies), $100 \mu \mathrm{g} / \mathrm{ml}$ heparin (Sigma-Aldrich, St. Louis, MO, USA) and $150 \mu \mathrm{g} / \mathrm{ml}$ endothelial cell growth supplement (Becton-Dickinson, Franklin Lakes, NJ, USA). For experiments, HUVEC between passages 3 and 5 were selected. The present study conformed to the ethical guidelines of the 1975 Declaration of Helsinki, with the approval of the Institutional Medical Ethics Committee of the Third Affiliated Hospital of Sun Yat-Sen University (Guangdong, China). All donors provided written informed consent. Human U937 monocytic cells were purchased from American Type Culture Collection (Manassas, VA, USA) and were cultured in RPMI-1640 medium (Invitrogen Life Technologies), supplemented with $15 \%$ fetal bovine serum and $100 \mathrm{U} / \mathrm{ml}$ penicillin-streptomycin. HUVEC and U937 cells were cultured at $37^{\circ} \mathrm{C}$ in a $5 \% \mathrm{CO}_{2}$ incubator with $90 \%$ humidity (Thermo Fisher Scientific, Waltham, MA, USA).

Adhesion assays. The U937 cells were labeled with $5 \mu \mathrm{M}$ calcein-acetoxymethyl ester (Invitrogen Life Technologies) for $30 \mathrm{~min}$ at $37^{\circ} \mathrm{C}$ in a $5 \% \mathrm{CO}_{2}$ incubator at $90 \%$ humidity (Thermo Fisher Scientific). The cells were subsequently washed twice with phosphate buffered saline (PBS; Invitrogen Life Technologies) and were resuspended in medium without serum. Following this, the cells were seeded onto confluent monolayers of HUVEC, which had been treated overnight with recombinant mouse tumor necrosis factor- $\alpha$ (TNF- $\alpha$; 20 ng/ml; PeproTech, Rocky Hill, NJ, USA). Following incubation for $1 \mathrm{~h}$ at $37^{\circ} \mathrm{C}$, the plates were rinsed twice with medium without serum. The adherent U937 cells remaining on the confluent monolayers of the HUVEC were counted using a fluorescence microscope (IX71; Olympus, Tokyo, Japan). For each condition, eight different visual fields (magnification, x200) in the middle of the dish were selected for analysis. HUVEC were pretreated with the $\mathrm{CX} 43$ channel inhibitor, oleamide $(50 \mu \mathrm{M})$, or enhancer, retinoic acid $(25 \mu \mathrm{M}$; Sigma-Aldrich), for different durations prior to adhesion assays and western blotting.

Overexpression of Cx43. Cx43 was expressed in HUVEC using a pcDNA3.1-Cx43 vector, kindly gifted by Professor Ryan Jensen and Professor Peter M. Glazer (Departments of Therapeutic Radiology and Genetics, Yale University School of Medicine, CT, USA). Transfection into the HUVEC was performed using Lipofectamine 2000 (Invitrogen Life Technologies), according to the manufacturer's instructions. The expression was assessed $72 \mathrm{~h}$ after transfection by western blotting.

Inhibition of Cx43 expression by small interfering (si)RNA transfection. An siRNA targeting the human $\mathrm{Cx} 43$ gene (5'-CAGUCUGCCUUUCGUUGUA-3') and a non-specific control siRNA were used (Invitrogen Life Technologies). For HUVEC, transfection with siRNA was performed at $50 \%$ confluence in 6 -well plates. A mixture of $50 \mathrm{nM}$ siRNA, Lipofectamine $2000(5 \mu \mathrm{l})$ and $500 \mu \mathrm{l}$ Opti-MEM (Invitrogen Life Technologies), were pre-incubated for $20 \mathrm{~min}$ at room temperature and the mixture was subsequently added to the 6 -well plates, containing 1,500 $\mu \mathrm{l}$ complete medium without antibiotics for $6 \mathrm{~h}$ in a $5 \% \mathrm{CO}_{2}$ incubator with $90 \%$ humidity. Following $72 \mathrm{~h}$ incubation, the knockdown efficiency was assessed by western blotting.

Western blotting. Western blotting was performed, as described previously (10). Whole-cell lysates for western blotting were prepared by washing the cells thrice with cell wash buffer [0.01 mol/1 PBS, $0.138 \mathrm{~mol} / 1 \mathrm{NaCl}, 0.02 \% \mathrm{NaN} 3$ (pH 7.4)] followed by a 2-h incubation in lysis buffer (Nanjing Keygen Biotech Co., Ltd., Nanjing, China) at $4^{\circ} \mathrm{C}$ using $0.05 \mathrm{ml} / \mathrm{cm}^{2}$. Protein concentrations were measured via the bicinchoninic acid method (Nanjing Keygen Biotech Co., Ltd.). Cell lysates $(10 \mu \mathrm{g})$ or purified connexin preparations $(10 \mu \mathrm{l})$ were separated by SDS-PAGE on 13\% Tris-glycine mini-gels (Invitrogen Life Technologies) and transferred onto polyvinylidene difluoride membrane (Bio-Rad Laboratories, Inc., Hercules, CA, USA). The membranes were blocked for $30 \mathrm{~min}$ at room temperature with $5 \%$ non fat dry milk (Sigma-Aldrich) and immunoblotted using anti-Cx43 antibody (1:3,000; mouse monoclonal Cx43 antibody raised against human; cat. no. C8093; Sigma-Aldrich) overnight at $4^{\circ} \mathrm{C}$. Following several washes, the membranes were incubated for $1 \mathrm{~h}$ at room temperature with anti-mouse horseradish peroxidase (HRP)-conjugated secondary antibodies $(1: 3,000$, goat polyclonal antibody raised against mouse IgG; cat. no. M6898; Sigma-Aldrich). The membranes were also incubated with anti-vascular cell adhesion molecule-1 (VCAM-1; mouse monoclonal VCAM-1 antibody raised against human; cat. no. sc-13160; Santa Cruz Biotechnology, Inc., Santa Cruz, CA, USA) and anti-intercellular cell adhesion molecule-1 (ICAM-1; mouse monoclonal ICAM-1 antibody raised against human; cat. no. sc-8439; Santa Cruz Biotechnology, Inc.) at a dilution of 1:200 overnight at $4^{\circ} \mathrm{C}$ and anti-mouse HRP-conjugated secondary antibodies (1:2,000; goat polyclonal antibody raised against mouse IgG; cat. no. M6898; Sigma-Aldrich) for $1 \mathrm{~h}$ at room temperature. Anti-Cx37 (goat polyclonal Cx37 antibody raised against 
A

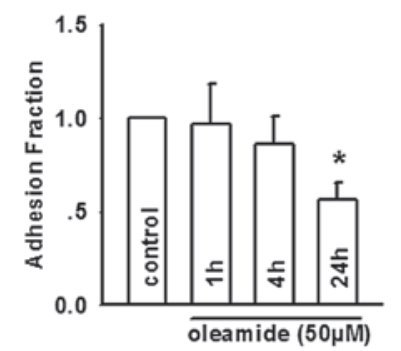

D

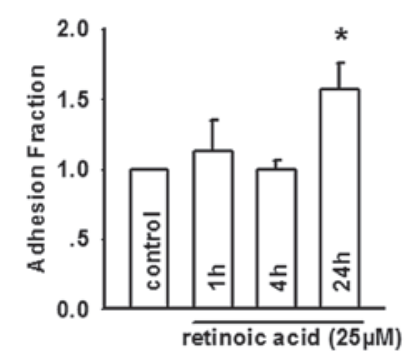

B

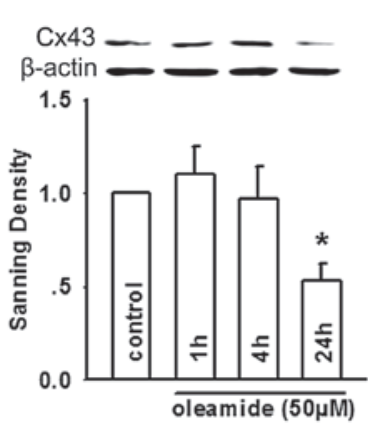

$\mathbf{E}$

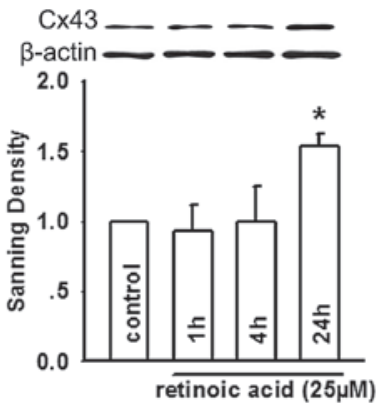

C

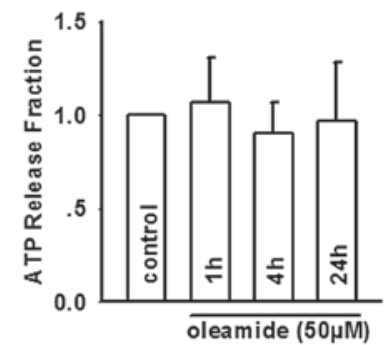

$\mathbf{F}$

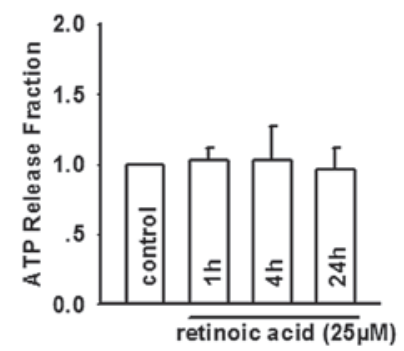

Figure 1. Effects of HUVEC exposure to oleamide and retinoic acid on monocyte-endothelial adhesion, expression of Cx43 and ATP release. The effects of treatment with (A-C) $50 \mu \mathrm{M}$ oleamide and (D-F) $25 \mu \mathrm{M}$ retinoic acid for 1, 4 or $24 \mathrm{~h}$ were investigated. The effects on the adhesion of HUVEC following treatment with (A) oleamide or (D) retinoic acid $\left(\mathrm{n}=5\right.$; $\left.{ }^{*} \mathrm{P}<0.01\right)$. The effects on the expression of $\mathrm{Cx} 43$ in HUVEC were assessed following treatment with (B) oleamide or (E) retinoic acid $\left(n=7\right.$; $\left.{ }^{\circ} \mathrm{P}<0.01\right)$. The effects on ATP release of the HUVEC was assessed following treatment (C) oleamide or (F) retinoic acid $(\mathrm{n}=6)$. HUVEC; human umbilical vein endothelial cells, ATP; adenosine triphosphate; Cx43, connexin 43.

human; cat. no. sc-27712; Santa Cruz Biotechnology, Inc.) and anti-Cx40 antibodies (mouse monoclonal Cx40 antibody raised against human; cat. no. sc-365107; Santa Cruz Biotechnology, Inc.) were both used at a dilution of 1:200. Secondary antibodies of Cx37 (1:2,000; donkey anti-goat IgG-HRP; cat. no. sc-2020; Santa Cruz Biotechnology, Inc.) was used at 1:1,000. Anti-mouse HRP-conjugated secondary antibodies of Cx40 (1:2,000; goat polyclonal antibody raised against mouse IgG; cat. no. M6898; Sigma-Aldrich) was used at 1:1,000. Mouse monoclonal anti- $\beta$-actin antibody raised against human (cat. no. A1978; Sigma-Aldrich) was used at a dilution of 1:10,000 and anti-mouse HRP-conjugated secondary antibodies (goat polyclonal antibody raised against mouse IgG; cat. no. M6898; Sigma-Aldrich) was used at 1:10,000. All primary antibodies were incubated overnight at $4^{\circ} \mathrm{C}$ and secondary antibodies were incubated for $1 \mathrm{~h}$ at room temperature. The protein bands were detected with an enhanced chemiluminescence system (KGP1125; Nanjing KeyGen Biotech. Co., Ltd.) Protein band sizes were estimated using Alpha View software (version 2.2.14407; ProteinSimple, Santa Clara, CA, USA).

Extracellular ATP measurements. Cellular ATP release was determined using an ATP bioluminescent assay kit (Sigma-Aldrich), according to the manufacturer's instructions (11). The supernatants of the HUVEC cultures were collected on ice. A total of $100 \mu \mathrm{l}$ supernatant was added to $100 \mu 1$ ATP assay mix solution. The luminescence was measured using a Cary Eclipse Fluorescence Spectrophotometer (FL0811M005; Bio/Chemi luminescence mode; Agilent Technologies, Palo Alto, CA, USA) in a 96-well culture plate.
Statistical analysis. Statistical analysis was performed using SPSS 15.0 software (SPSS, Inc., Chicago, IL, USA). Multiple comparisons among groups were analyzed using one-way analysis of variance, followed by Tukey post hoc comparisons. $\mathrm{P}<0.05$ was considered to indicate a statistically significant difference.

\section{Results}

Effects of oleamide and retinoic acid treatment on the expression of CX43, ATP release and U937-HUVEC adhesion in $H U V E C$. To investigate the role of HUVEC connexins on monocyte-endothelial adhesion, HUVEC were treated with oleamide, which rapidly inhibited $\mathrm{Cx}$ channels, including Cx43 (14), or with retinoic acid, which our previous study demonstrated increases the expression of $\mathrm{Cx} 43$ in these cells (15). The effects of oleamide and retinoic acid treatment on U937-HUVEC adhesion, Cx43 expression and ATP release were determined. As shown in Fig. 1A, oleamide $(50 \mu \mathrm{M})$ revealed no rapid effect on adhesion, however, did modestly attenuate adhesion at $24 \mathrm{~h}$, which correlated with a decrease in the expression of $\mathrm{Cx} 43$ in HUVEC (Fig. 1B). Previous studies suggest that a rapid effect on adhesion is always associated with ATP release, particularly from monocytes, which inhibits monocyte-endothelial adhesion, and the depression of ATP release may thereby enhance atherosclerotic progression $(11,16)$. These studies suggested that ATP release can markedly affect cell adhesion. Therefore, the present study detected ATP release from HUVEC exposed to oleamide. No effects were observed on ATP release (Fig. 1C). This suggested that in HUVEC, Cx43 channel activity was not involved 
A

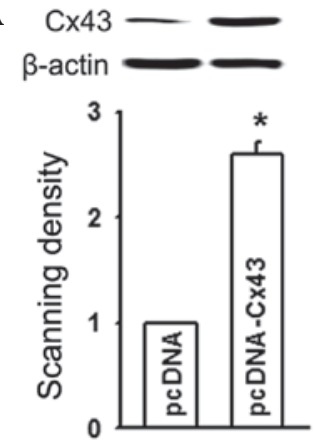

D $\underset{\beta-a c t i n}{\mathrm{C} 43}=$

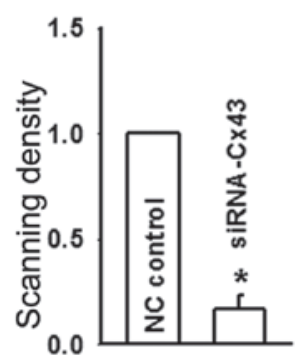

B

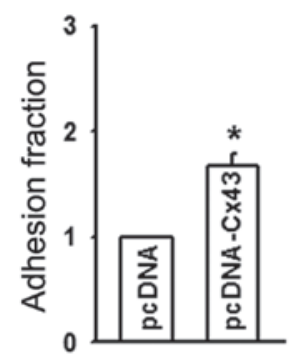

$\mathbf{E}$

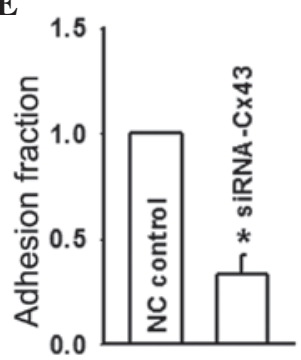

C

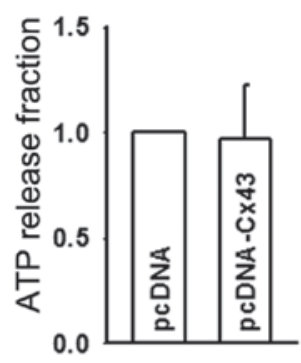

F

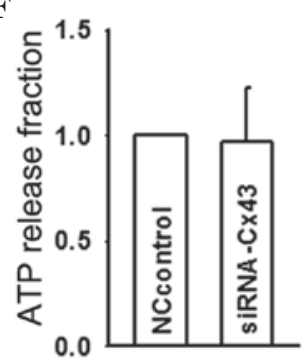

Figure 2. Expression of $\mathrm{Cx} 43$ in HUVEC regulates monocyte-endothelial adhesion, however, reveals no affect on ATP release. (A) The expression of Cx43 in HUVEC was assessed following transfection with pcDNA-Cx43 $\left(\mathrm{n}=5\right.$; $\left.{ }^{*} \mathrm{P}<0.05\right)$. (B) Monocyte-endothelial adhesion increased when $\mathrm{Cx} 43$ was overexpressed in HUVEC $\left(n=5 ;{ }^{*} \mathrm{P}<0.05\right)$. (C) The release of ATP from HUVEC remained unchanged when the expression of $\mathrm{Cx} 43$ was increased with pcDNA-Cx43 ( $\mathrm{n}=7$ ). (D) The expression of Cx43 in HUVEC was assessed following treatment with siRNA-Cx43 ( $n=5$; $\left.{ }^{*} \mathrm{P}<0.05\right)$. (E) Monocyte-endothelial adhesion decreased when $\mathrm{Cx} 43$ was knocked down with siRNA-Cx43 $\left(\mathrm{n}=4 ;{ }^{*} \mathrm{P}<0.05\right)$. (F) The release of ATP from HUVEC remained unchanged when the expression of $\mathrm{Cx} 43$ was decreased by siRNA-Cx43 (n=7). HUVEC; human umbilical vein endothelial cells, ATP; adenosine triphosphate; Cx43, connexin 43; si, small interfering; $\mathrm{NC}$, negative control.

in adhesion, however, a decrease in Cx43 expression may attenuate it. To further characterize the association between the expression of $\mathrm{Cx} 43$ and U937-HUVEC cell adhesion, the effects of retinoic acid, a commonly used potentiator of $\mathrm{Cx}$ expression and function was assessed. Treatment of HUVEC with retinoic acid $(25 \mu \mathrm{M})$ induced increased expression of $\mathrm{Cx} 43$ at $24 \mathrm{~h}$, however, not at shorter durations, which correlated with increased adhesion (Fig. 1D and E). ATP release from HUVEC exposed to retinoic acid remained unchanged (Fig. 1F). These findings suggested that U937-HUVEC cell adhesion was positively modulated by the expression of Cx43 in HUVEC, and was essentially unaffected by altering of the $\mathrm{Cx} 43$ function.

Direct modulation of the expression of CX43 in HUVEC modulates U937-HUVEC adhesion. To more directly and specifically establish the role of Cx43 in U937-HUVEC adhesion, the expression of $\mathrm{Cx} 43$ was specifically altered by two methods: Over-expression by transfection with pcDNA-Cx43 and knock-down using siRNA-Cx43. As shown in Fig. 2A and B, Cx43 expression in HUVEC was enhanced nearly two-fold following pcDNA-Cx43-transfection, and this markedly increased adhesion to monocytes. However, ATP release from HUVEC remained unaffected by pcDNA-Cx43-transfection, which correlated with HUVEC exposed to the $\mathrm{Cx} 43$ potentiator, retinoic acid (Figs. $1 \mathrm{C}$ and $2 \mathrm{C}$ ). Fig. 2D and $\mathrm{E}$ demonstrated that the expression of $\mathrm{Cx} 43$ was downregulated by siRNA-Cx43 and this led to a decrease in adhesion, however, ATP release from HUVEC remained unaf-
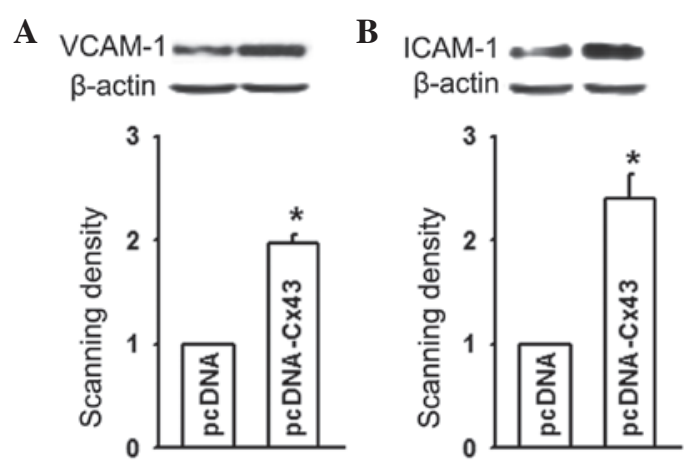

C

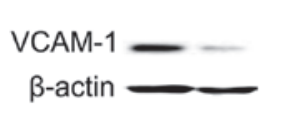

${ }_{\beta \text { ICAM-1 }}^{\text {ICactin }}=$
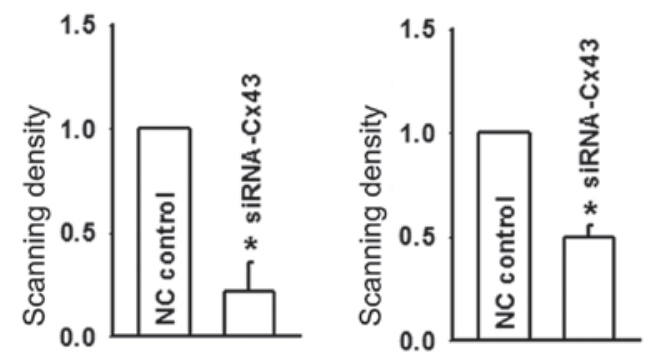

Figure 3. Altered expression of $\mathrm{Cx} 43$ regulates the expression levels of VCAM-1 and ICAM-1. (A and B) Cx43 overexpression in HUVEC by pcDNA-Cx43 increased the expression levels of VCAM-1 and ICAM-1 ( $n=5$; ${ }^{*} \mathrm{P}<0.05$ ). (C and D) Knockdown of Cx43 by siRNA-Cx43 attenuated the expression levels of VCAM-1 and ICAM-1 $\left(n=5 ;{ }^{*} \mathrm{P}<0.05\right)$. HUVEC; human umbilical vein endothelial cells; $\mathrm{Cx} 43$, connexin 43; si, small interfering; $\mathrm{NC}$, negative control. 
A

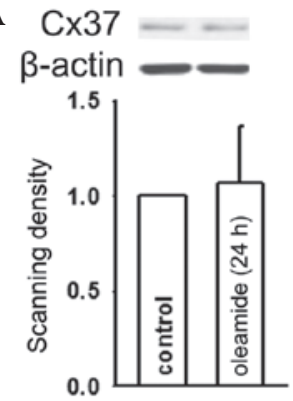

C

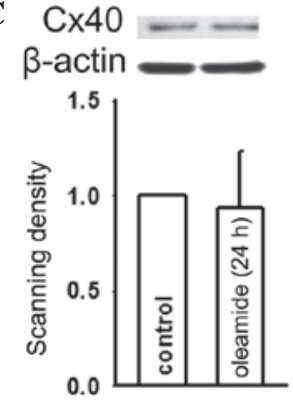

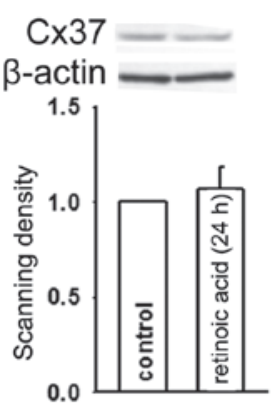
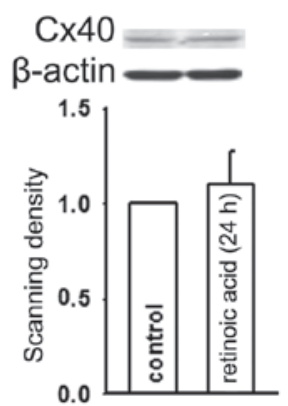

B

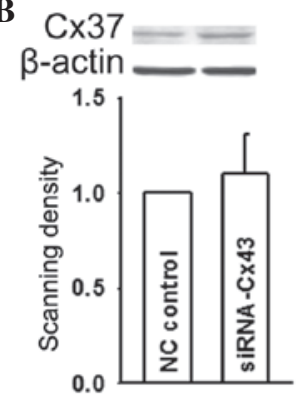

D $\underset{\beta-\operatorname{actin}}{\mathrm{C} 40}=$

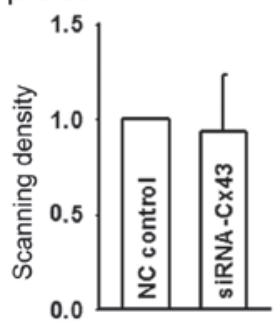

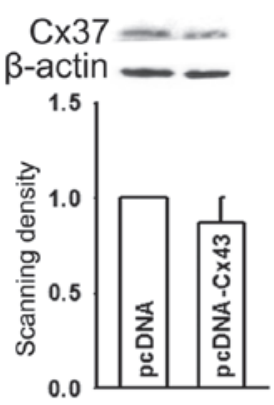

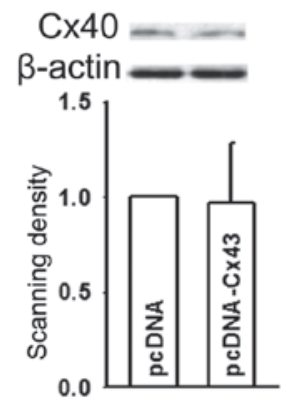

Figure 4. Altered expression of $\mathrm{Cx} 43$ reveals no affect on the expression levels of Cx37 and Cx40. The expression of Cx37 remained unchanged when (A) HUVEC were exposed to oleamide $(50 \mu \mathrm{M})$ or retinoic acid $(25 \mu \mathrm{M})$ for $24 \mathrm{~h}\left(\mathrm{n}=4\right.$; $\left.{ }^{*} \mathrm{P}<0.05\right)$ and $(\mathrm{B})$ when the expression of $\mathrm{Cx} 43$ was altered by transfection with pcDNA-Cx43 or by siRNA-Cx43 $\left(\mathrm{n}=4 ;{ }^{*} \mathrm{P}<0.05\right)$. The expression of $\mathrm{Cx} 40$ remained unchanged when (C) HUVEC were exposed to oleamide $(50 \mu \mathrm{M})$ or retinoic acid $(25 \mu \mathrm{M})$ for $24 \mathrm{~h}\left(\mathrm{n}=4 ;{ }^{*} \mathrm{P}<0.05\right)$ and $(\mathrm{D})$ when $\mathrm{Cx} 43$ expression was altered by transfection with pcDNA-Cx43 or by siRNA-Cx43 ( $=4$; $\left.{ }^{*} \mathrm{P}<0.05\right)$. HUVEC; human umbilical vein endothelial cells; $\mathrm{Cx}$, connexin; si, small interfering.

fected (Fig. 2F). These results, in combination with those in Fig. 1, demonstrated that even though $\mathrm{Cx} 43$ channel function in HUVEC revealed no effect on adhesion, the expression of Cx43 in HUVEC positively regulated it.

Direct modulation of the expression of CX43 regulates cell adhesion proteins, VCAM-1 and ICAM-1, in HUVEC. The cell adhesion proteins, VCAM-1 and ICAM-1, belong to the immunoglobulin superfamily, whose endothelial expression is closely associated with the development of atherosclerosis (17). Since the results shown in Figs. 1 and 2 indicated that the expression of $\mathrm{Cx} 43$ in HUVEC positively regulated U937-HUVEC cell adhesion, the present study next determined whether alteration of the expression of $\mathrm{Cx} 43$ in HUVEC affected the expression levels of VCAM-1 and ICAM-1. The results demonstrated that when Cx43 expression was increased by pcDNA-Cx43-transfection, VCAM-1 and ICAM-1 expression were markedly upregulated (Fig. 3A and B). By contrast, when the expression of $\mathrm{Cx} 43$ was reduced by siRNA-Cx43 in HUVEC, the expression levels of VCAM-1 and ICAM-1 declined as the expression of Cx43 decreased (Fig. 3C and D). This suggested that the expression of $\mathrm{Cx} 43$ in HUVEC promoted cell adhesion by increasing the expression of VCAM-1 and ICAM-1.

Altering the expression of CX43 in HUVEC reveals no effect on the expression levels of Cx37 or Cx40. Previous studies demonstrated three different types of connexins expressed in HUVEC, Cx37, Cx40 and Cx43 (18-20). It was previously demonstrated that $\mathrm{Cx} 37$ promotes ATP release, which reduced monocyte adhesion to endothelial cells, thereby interfering with atherosclerotic development $(8,9)$. It was also demonstrated that $\mathrm{Cx} 40$-mediated gap junction communication contributed to the attenuation of leukocyte adhesion to the endothelium $(8,9)$. In order to exclude the effects of $\mathrm{Cx} 37$ and Cx40 on cell adhesion, the present study assessed the expression levels of $\mathrm{Cx} 37$ and $\mathrm{Cx} 40$ when $\mathrm{Cx} 43$ expression was altered. Fig. 1 demonstrated that at $24 \mathrm{~h}$, the expression of Cx43 was markedly changed along with cell adhesion when HUVEC were exposed to oleamide or retinoic acid. However, the expression of $\mathrm{Cx} 37$ revealed no change under identical conditions (Fig. 4A). Notably, when Cx43 in HUVEC was overexpressed by transfection with pcDNA-Cx43 or knocked down with siRNA-Cx43, Cx37 expression remained unchanged (Fig. 4B). An identical result was obtained for $\mathrm{Cx} 40$ (Fig. 4C and D). These results indicated that $\mathrm{Cx} 43$ expression, which regulated cell adhesion, was independent of the expression levels of $\mathrm{Cx} 37$ or $\mathrm{Cx} 40$ in HUVEC.

\section{Discussion}

The present study investigated the effect of $\mathrm{Cx} 43$ function and expression in vascular endothelial cells on monocyte-endothelial adhesion, the initial step in atherosclerotic development. It was confirmed that in HUVEC, upregulation of the expression of Cx43 enhanced cell adhesion and downregulation markedly decreased cell adhesion. Notably, two different types of crucial cell adhesion protein, VCAM-1 and ICAM-1, were affected by changes in the expression of $\mathrm{Cx} 43$, which mediated the recruitment of circulating monocytes onto endothelial cells. A previous study demonstrated that ATP release is important in monocyte-endothelial adhesion, particularly from Cx37 chan- 
nels, which contributed to a rapid decrease in adhesion (8). However, the possible contribution of $\mathrm{Cx} 43$ in HUVEC remains to be elucidated. It has been well documented that $\mathrm{Cx} 43$ is impermeable to ATP in monocytes, which may modulate monocyte-endothelial adhesion (11), however, in the present study, it was revealed that ATP release from HUVEC revealed no effect on cell adhesion and that monocyte-endothelial adhesion may be regulated by changes in the expression of $\mathrm{Cx} 43$ modulating the cell adhesion proteins, VCAM-1 and ICAM-1.

Downregulation of $\mathrm{Cx} 43$ had been demonstrated to downregulate the expression of VCAM-1 in other systems (21). The specific mechanism remains to be elucidated, however, there were several likely pathways. One pathway involves interactions between the carboxyl-terminal domain of $\mathrm{Cx} 43$ and elements of various cellular signaling pathways, including cytoskeletal components, Src, PKA and PKC, which have downstream effects on cell adhesion molecules (22). In particular, it is known that the expression levels of VCAM-1 and ICAM-1 are closely associated with the activation of $\mathrm{NF}-\kappa \mathrm{B}$ and $\mathrm{PKC}$, and that $\mathrm{Cx} 43$ amplifies the intercellular signaling, which activates NF- $\mathrm{B}$ (23).

Several previous studies revealed that $\mathrm{Cx}$ hemichannels release cytosolic compounds, including ATP (8). ATP release through hemichannels may be dynamically regulated by several factors, including changes in intracellular $\mathrm{Ca}^{2+}$, ischaemic stress, hypoxia and, important in the context of atherosclerosis, cytokines $(24,25)$. Atherosclerosis is an inflammatory disease in which the release of cytokines is prominent. Cytokines, including TNF- $\alpha$, interleukin, IFN- $\gamma$ and TGF- $\beta$, inhibit the activity of $\mathrm{Cx} 43$ channels $(26,27)$. Therefore, cytokine release in atherosclerotic progression may attenuate ATP release from monocytes, enhancing adhesion in a positive-feedback inflammatory cycle. Although $\mathrm{Cx} 43$ was expressed by U937 and HUVEC, it was notable that Cx43 hemichannel activity appeared to be differentially regulated in the two cell types. In our previous study, monocyte-endothelial adhesion was revealed to be modulated by $\mathrm{Cx} 43$ through ATP release in U937 cells (11). However, in the present study, Cx43 in HUVEC did not exert this function. This difference may arise from interactions of the carboxyl-terminal domain of Cx43 with different regulatory binding partners and signaling pathways in the two cell types (28).

To date, three different types of connexins, Cx37, Cx40 and $\mathrm{Cx} 43$, have been revealed to be expressed in HUVEC, which may influence monocyte-endothelial adhesion and protect against atherosclerosis through various mechanisms $(8,9)$. In the present study, four distinct methods were used to assess the effects of $\mathrm{Cx} 43$ on monocyte-endothelial adhesion: Inhibition using oleamide; enhancement with retinoic acid; overexpression of $\mathrm{Cx} 43$ by transfection with pcDNA-Cx43 and knock-down of $\mathrm{Cx} 43$ expression with siRNA-Cx43. The results revealed that $\mathrm{Cx} 43$ expressed in HUVEC influenced monocyte-endothelial adhesion by modulating the expression levels of cell adhesion proteins, VCAM-1 and ICAM-1. The upregulation of Cx43 expression enhanced monocyte-endothelial adhesion and downregulation of Cx43 markedly decreased it. In order to confirm the specific function of $\mathrm{Cx} 43$ on monocyte-endothelial adhesion, whether modulation of $\mathrm{Cx} 43$ was dependent on $\mathrm{Cx} 37$ or $\mathrm{Cx} 40$ was investigated. Fig. 4 illustrated that neither $\mathrm{Cx} 37$ nor
Cx40 were affected by the altered expression of $\mathrm{Cx} 43$, which indicated that the effects of Cx43 expression in HUVEC on monocyte-endothelial adhesion were specific and distinctive, independent of $\mathrm{Cx} 37$ or $\mathrm{Cx} 40$.

The role of the expression and/or function of $\mathrm{Cx} 43$ in the pathogenesis of atherosclerosis were complex, suggesting various roles in different phases of atherosclerosis. For example, Cx43 modulated monocyte migration to the arterial intima through the interaction between the carboxyl-terminal tails of Cx43 and microtubules (29,30). These subsequently became foam cells in arterial intima and this process was also regulated by the expression of Cx43 (12). The expression of Cx43 was always markedly increased at the curved areas or the branch points of large arteries and the oscillatory shear stress in those locations, which contributed to atherosclerotic plaques formation (31). Reduced endothelial expression of Cx43 was associated with plaque stability (13).

The present study focused on the initiating step of atherosclerosis, monocyte-endothelial adhesion. However, the present study differed compared with our previous investigation, which focused on the expression of $\mathrm{Cx} 43$ in monocytes and the effects of endothelial cells expressing Cx43 on adhesion. The present findings, along with our previous conclusion, suggested that altered expression of $\mathrm{Cx} 43$ in monocytes and endothelial cells may regulate cell adhesion. Notably, it was determined that Cx43 channel-stimulated ATP release in monocytes can affect rapid adhesion, however, the expression of Cx43 in HUVEC did not exert this function. The differences described here offer distinct basis for targeted intervention.

\section{Acknowledgements}

This study was supported by the National Natural Science Foundation of China (nos. 81170449 and 81401628), the key project of Natural Science Foundation of Guangdong Province, China (no. S2011020002780) and the Medical Research Foundation of Guangdong Province (no. B2014141).

\section{References}

1. Burnier L, Fontana P, Angelillo-Scherrer A and Kwak BR: Intercellular communication in atherosclerosis. Physiology (Bethesda) 24: 36-44, 2009.

2. Boon RA and Horrevoets AJ: Key transcriptional regulators of the vasoprotective effects of shear stress. Hamostaseologie 29: 39-43, 2009.

3. Binder CJ, Chang MK, Shaw PX, Miller YI, Hartvigsen K, Dewan A and Witztum JL: Innate and acquired immunity in atherogenesis. Nat Med 8: 1218-1226, 2002.

4. Sumiyoshi A: Pathological processes of coronary atherosclerosis. Nihon Rinsho (Suppl 52): S85-S89, 1994 (In Japanese).

5. Rebrov AP and Voskoboi IV: Effect of inflammatory and infectious factors in the development of atherosclerosis. Ter Arkh 76: 78-82, 2004 (In Russian).

6. Insull W Jr: The pathology of atherosclerosis: Plaque development and plaque responses to medical treatment. Am J Med 122 (Suppl 1): S3-S14, 2009

7. Tzoulaki I, Murray GD, Price JF, Smith FB, Lee AJ, Rumley A Lowe GD and Fowkes FG: Hemostatic factors, inflammatory markers and progressive peripheral atherosclerosis: The Edinburgh Artery Study. Am J Epidemiol 163: 334-341, 2006.

8. Wong CW, Christen T, Roth I, Chadjichristos CE, Derouette JP, Foglia BF, Chanson M, Goodenough DA and Kwak BR: Connexin37 protects against atherosclerosis by regulating monocyte adhesion. Nat Med 12: 950-954, 2006. 
9. Chadjichristos CE, Scheckenbach KE, van Veen TA, Richani Sarieddine MZ, de Wit C, Yang Z, Roth I, Bacchetta M, Viswambharan H, Foglia B, et al: Endothelial-specific deletion of connexin 40 promotes atherosclerosis by increasing CD73-dependent leukocyte adhesion. Circulation 121: 123-131, 2010.

10. Luo C, Yuan D, Li X, Yao W, Luo G, Chi X, Li H, Irwin MG, $\mathrm{Xia} \mathrm{Z}$ and Hei Z: Propofol attenuated acute kidney injury after orthotopic liver transplantation via inhibiting gap junction composed of connexin 32. Anesthesiology 122: 72-86, 2015.

11. Lee ES, Van Spyk EN, Chun KC, Pitts RL, Wu MH and Yuan SY: Monocytic adhesion molecule expression and monocyte-endothelial cell dysfunction are increased in patients with peripheral vascular disease versus patients with abdominal aortic aneurysms. J Surg Res 177: 373-381, 2012.

12. Derouette JP, Wong C, Burnier L, Morel S, Sutter E, Galan K, Brisset AC, Roth I, Chadjichristos CE and Kwak BR: Molecular role of $\mathrm{Cx} 37$ in advanced atherosclerosis: A micro-array study. Atherosclerosis 206: 69-76, 2009.

13. Wong CW, Burger F, Pelli G, Mach F and Kwak BR: Dual benefit of reduced $\mathrm{Cx} 43$ on atherosclerosis in LDL receptor-deficient mice. Cell Commun Adhes 10: 395-400, 2003.

14. Ransjö M, Sahli J and Lie A: Expression of connexin 43 mRNA in microisolated murine osteoclasts and regulation of bone resorption in vitro by gap junction inhibitors. Biochem Biophys Res Commun 303: 1179-1185, 2003.

15. Tanmahasamut $P$ and Sidell N: Up-regulation of gap junctional intercellular communication and connexin43 expression by retinoic acid in human endometrial stromal cells. J Clin Endocrinol Metab 90: 4151-4156, 2005.

16. Eltzschig HK, Eckle T, Mager A, Küper N, Karcher C, Weissmüller T, Boengler K, Schulz R, Robson SC and Colgan SP: ATP release from activated neutrophils occurs via connexin43 and modulates adenosine-dependent endothelial cell function. Circ Res 99: 1100-1108, 2006.

17. Fotis L, Agrogiannis G, Vlachos IS, Pantopoulou A, Margoni A Kostaki M, Verikokos C, Tzivras D, Mikhailidis DP and Perrea D: Intercellular adhesion molecule (ICAM)-1 and vascular cell adhesion molecule (VCAM)-1 at the early stages of atherosclerosis in a rat model. In Vivo 26: 243-250, 2012.

18. Dhein S, Gaertner C, Georgieff C, Salameh A, Schlegel F and Mohr FW: Effects of isoprenaline on endothelial connexins and angiogenesis in a human endothelial cell culture system. Naunyn Schmiedebergs Arch Pharmacol 388: 101-108, 2015.

19. Godecke S, Roderigo C, Rose CR, Rauch BH, Godecke A and Schrader J: Thrombin-induced ATP release from human umbilical vein endothelial cells. Am J Physiol Cell Physiol 302: C915-C923, 2012.
20. Haussig S, Schubert A, Mohr FW and Dhein S: Sub-chronic nicotine exposure induces intercellular communication failure and differential down-regulation of connexins in cultured human endothelial cells. Atherosclerosis 196: 210-218, 2008.

21. Toubas J, Beck S, Pageaud AL, Huby AC, Mael-Ainin M, Dussaule JC, Chatziantoniou $\mathrm{C}$ and Chadjichristos $\mathrm{CE}$ : Alteration of connexin expression is an early signal for chronic kidney disease. Am J Physiol Renal Physiol 301: F24-F32, 2011.

22. Giepmans BN: Gap junctions and connexin-interacting proteins. Cardiovasc Res 62: 233-245, 2004.

23. Martin FJ and Prince AS: TLR2 regulates gap junction intercellular communication in airway cells. J Immunol 180: 4986-4993, 2008.

24. Faigle M, Seessle J, Zug S, El Kasmi KC and Eltzschig HK: ATP release from vascular endothelia occurs across $\mathrm{Cx} 43$ hemichannels and is attenuated during hypoxia. PLoS One 3: e2801, 2008.

25. Anselmi F, Hernandez VH, Crispino G, Seydel A, Ortolano S, Roper SD, Kessaris N, Richardson W, Rickheit G, Filippov MA, et al: ATP release through connexin hemichannels and gap junction transfer of second messengers propagate $\mathrm{Ca}^{2+}$ signals across the inner ear. Proc Natl Acad Sci USA 105: 18770-18775, 2008.

26. Lim MC, Maubach G and Zhuo L: TGF-betal down-regulates connexin43 expression and gap junction intercellular communication in rat hepatic stellate cells. Eur J Cell Biol 88: 719-730, 2009.

27. Kimura $\mathrm{K}$ and Nishida T: Role of the ubiquitin-proteasome pathway in downregulation of the gap-junction protein Connexin 43 by TNF- alpha\} in human corneal fibroblasts. Invest Ophthalmol Vis Sci 51: 1943-1947, 2010.

28. Laird DW: The gap junction proteome and its relationship to disease. Trends Cell Biol 20: 92-101, 2010.

29. Rhee DY, Zhao XQ, Francis RJ, Huang GY, Mably JD and Lo CW: Connexin43 regulates epicardial cell polarity and migration in coronary vascular development. Development 136: 3185-3193, 2009

30. Behrens J, Kameritsch P, Wallner S, Pohl U and Pogoda K: The carboxyl tail of $\mathrm{Cx} 43$ augments p38 mediated cell migration in a gap junction-independent manner. Eur J Cell Biol 89: 828-838, 2010.

31. Inai T, Mancuso MR, McDonald DM, Kobayashi J, Nakamura K and Shibata Y: Shear stress-induced upregulation of connexin43 expression in endothelial cells on upstream surfaces of rat cardiac valves. Histochem Cell Biol 122: 477-483, 2004. 